\title{
Long-Ranged Oppositely Charged Interactions for Designing New Types of Colloidal Clusters
}

\author{
Ahmet Faik Demirörs, ${ }^{*}$ Johan C. P. Stiefelhagen, Teun Vissers, ${ }^{\dagger}$ Frank Smallenburg, ${ }^{\ddagger}$ \\ Marjolein Dijkstra, Arnout Imhof, and Alfons van Blaaderen ${ }^{\S}$ \\ Department of Physics, Soft Condensed Matter, Debye Institute for Nanomaterials Science, \\ Utrecht University, Princetonplein 5, 3584 CC Utrecht, Netherlands
}

(Received 23 October 2014; published 29 April 2015)

\begin{abstract}
Getting control over the valency of colloids is not trivial and has been a long-desired goal for the colloidal domain. Typically, tuning the preferred number of neighbors for colloidal particles requires directional bonding, as in the case of patchy particles, which is difficult to realize experimentally. Here, we demonstrate a general method for creating the colloidal analogs of molecules and other new regular colloidal clusters without using patchiness or complex bonding schemes (e.g., DNA coating) by using a combination of long-ranged attractive and repulsive interactions between oppositely charged particles that also enable regular clusters of particles not all in close contact. We show that, due to the interplay between their attractions and repulsions, oppositely charged particles dispersed in an intermediate dielectric constant $(4<\varepsilon<10)$ provide a viable approach for the formation of binary colloidal clusters. Tuning the size ratio and interactions of the particles enables control of the type and shape of the resulting regular colloidal clusters. Finally, we present an example of clusters made up of negatively charged large and positively charged small satellite particles, for which the electrostatic properties and interactions can be changed with an electric field. It appears that for sufficiently strong fields the satellite particles can move over the surface of the host particles and polarize the clusters. For even stronger fields, the satellite particles can be completely pulled off, reversing the net charge on the cluster. With computer simulations, we investigate how charged particles distribute on an oppositely charged sphere to minimize their energy and compare the results with the solutions to the well-known Thomson problem. We also use the simulations to explore the dependence of such clusters on Debye screening length $\kappa^{-1}$ and the ratio of charges on the particles, showing good agreement with experimental observations.
\end{abstract}

DOI: 10.1103/PhysRevX.5.021012

Subject Areas: Materials Science, Physical Chemistry, Soft Matter

\section{INTRODUCTION}

Colloidal self-assembly is crucial for bottom-up fabrication of new functional materials and devices. However, the range of colloidal crystals that can be created with common spherical colloids with isotropic interactions is limited. This range can be expanded by using colloids that

\footnotetext{
*Present address: Department of Materials, ETH Zurich, 8093 Zurich, Switzerland.

A.F.Demirors@gmail.com

${ }^{\dagger}$ Present address: School of Physics and Astronomy, The University of Edinburgh, Kings Buildings, Peter Guthrie Tait Road, Edinburgh, EH9 3FD, United Kingdom.

${ }^{\star}$ Present address: Institut für Theoretische Physik II-Soft Matter Heinrich-Heine-Universität Düsseldorf, Düsseldorf, Germany.

${ }^{\S}$ A.vanBlaaderen@uu.nl

Published by the American Physical Society under the terms of the Creative Commons Attribution 3.0 License. Further distribution of this work must maintain attribution to the author(s) and the published article's title, journal citation, and DOI.
}

have nonspherical shapes or interaction potentials [1-7]. Nature uses highly anisotropic building blocks, such as proteins, to self-assemble intricate ternary and quaternary functional complexes. Achieving a similar level of sophistication using colloids could open a world of exciting applications of which a full three-dimensional photonic band gap material is just one example [8]. Furthermore, packings of these anisotropic colloids with symmetries found in molecules, which have recently been called "colloidal molecules" [2,9-12], can provide a useful model for understanding molecular crystallization and can also give insight into self-assembled devices from anisotropic particles [13]. In addition, the binary nature of the clusters may help to design metamaterials with controlled properties that arise from the organization and the stoichiometry of the components $[14,15]$.

Binary colloidal crystals have significantly increased the number of different types of colloidal crystals that can be realized experimentally, and in addition, the close connection of particles that have different properties opens many different avenues to tailor new materials properties 
[14-18]. Creating regular colloidal clusters of particles with different properties will increase possibilities for both the single clusters and materials self-assembled from them in similar ways. In the following, we give a brief and, therefore, incomplete overview of methods already developed in the past decade to make clusters of mostly spherical particles. We limit ourselves here somewhat arbitrarily to methods that focus on regular clusters from $n<20$ building blocks. One of the most used methods is the one developed by the Pine group [10], in which particles associated with the surface of emulsion droplets are selfassembled by the drying of the emulsion. It has been found that the complex drying forces of such clusters minimize the second moment of the mass distribution of the clusters [19]. Several methods make use, in some way or another, of the polymerization of organic monomers associated with spherical particles $[1,12,20-23]$. The methodologies include swelling of already existing cross-linked particles (for an example from our own group, see Ref. [20] and the references cited therein), aggregation of liquid protrusions of a monomer [21,23], but also wetting of an organic monomer on inorganic seed particles [11]. Another approach is using patchy interactions on spherical particles [24]. Making clusters of anisotropic particles using these methodologies has also just started; see, e.g., Refs. [22,25] for examples using dumbbells and nanorods.

More recently, methods have been developed that more explicitly use the interplay between attractions and repulsions between the particles to create clusters of a relatively small number of particles; see, e.g., Refs. [7,26-29]. The attractions can be, for instance, caused by depletion effects or between particles of opposite charge. Next to the attractions there also needs to be an effective repulsive part in the cluster-cluster interactions to keep the clusters stable, unless each cluster is made in isolation [16]. In this paper, we focus on using oppositely charged interactions with, in addition, a Debye-Hückel screening length that is relatively large compared to the particle sizes used in the cluster generation. Our work is motivated by and partially uses systems of oppositely charged particles that our group $[17,18,30]$ and others independently of us [31] found to be able to produce equilibrium crystallization of oppositely charged spherical particles.

Heteroaggregation of a relatively small number of large seed particles by small particles of opposite charge has already been used by several groups, see, e.g., Refs. [32-37], for instance, to make core-shell systems by heating [35], to incorporate layers of the smaller particles in polyelectrolyte layers [36], or to induce a specific roughness on the seed particles to tune depletion forces [37]. It is also well known that if the double layer becomes the size of the particles or larger, then more linear structures are formed, although experiments in this limit are relatively rare [38-42]. Heteroaggregation was frequently studied to test theories on the interactions between oppositely charged particles
[43] and/or the fractal nature of the resulting 3D gels and aggregates [40,44]. However, as mentioned, it is only recently that groups have started to explore heteroaggregation as a way to arrive at regular clusters of a small number of oppositely charged building blocks $[7,27,29,45]$.

In this work, we demonstrate a general bulk method for creating the colloidal analogs of molecules out of isotropic building blocks, based on the attractive and repulsive interactions between oppositely charged particles with double layers of the particle size or larger. Under the right circumstances, mixing positively and negatively charged particles at low concentration causes them to self-assemble into clusters consisting of one central particle (labeled $A$ ) with a small number of (oppositely charged) satellite (labeled $B$ ) particles attached to its surface. The clusters are stabilized against further aggregation by their remaining net charge and the large extension of the double layer, and persist on macroscopic time scales. Furthermore, the (intracluster) repulsions between the satellite particles lead to well-defined preferences for the position and number of satellites on each central particle that differ from other methodologies to make small clusters. We show fabrication of these colloidal molecules in two different experimental systems, demonstrating that our method can be adapted to different materials. As equilibrium crystallization between oppositely charged particles has already been extended to nanoparticle systems [15], and quite recently also to watersoluble particles [46], we expect the methods developed in this paper to also be extendable to these systems as well. In addition to fabricating colloidal analogs of molecules, we also show that the stability and interactions of clusters can be altered with an external electric field; see also Ref. [17]. To complement and better understand the experiments, we perform Monte Carlo simulations for the minimum-energy structure of individual clusters. For unscreened charges, this problem reduces to the well-known Thomson problem [47], but charge screening changes the ground state for several cluster sizes.

\section{RESULTS AND DISCUSSION}

\section{A. Experimental results}

To demonstrate the fabrication of regular binary colloidal clusters experimentally, we use oppositely charged particles in an intermediate dielectric constant $(4<\varepsilon<10)$ solvent, where colloids have long-ranged repulsive interactions $[48,49]$ with Debye screening lengths on the order of a few particle diameters. In such an apolar solvent, the concentration of ions is low, but nonzero, such that the electric potential of each particle is screened. This alters a simple Coulomb potential into a screened Coulomb potential, also known as a Yukawa potential. Because of their attractive interactions, under the right conditions oppositely charged particles can self-assemble into small aggregates, consisting of a (for example) positively charged central 
particle surrounded by a small number of oppositely charged satellite particles. These clusters are stabilized against further aggregation by their net charge and longranged repulsion, leading to a large distance (several particle diameters) between individual clusters [48,49]. The resulting system is a mixture of clusters and possibly excess free satellite particles. The structure of the cluster is determined by the repulsion between the satellite particles, yielding highly symmetric moleculelike constructions. Four conditions appear to be necessary for regular cluster formation: (i) the satellite particles need to have sufficient repulsions between each other, (ii) after the cluster is formed, the net charge on the cluster has to be high enough to repel other clusters such that clusters do not coagulate, (iii) there has to be an excess of satellite particles, i.e., a number ratio $N_{B}: N_{A}$ larger than $3: 1$, to prevent particles from simply forming a gel consisting of a continuous network of alternating positive and negative particles $[41,42,44]$, and finally, (iv) there have to be attractions between the oppositely charged species that should be larger than $\sim 10 \mathrm{kT}$, so that the clusters remain fixed. Once a particle has been surrounded by an optimal number of particles of the opposite charge, the scarcity of the core particles results in separate clusters rather than larger aggregates.

In our experiments, we use mixtures of oppositely charged polymethylmethacrylate (PMMA) colloids, mixtures of positively charged titania and negatively charged silica colloids, and mixtures of oppositely charged silica colloids. Oppositely charged PMMA colloids are dispersed in a mixture of $72.8 \%$ cyclohexyl bromide (CHB) and $27.2 \%$ cis-decalin (cis-dec) $[\varepsilon=5.62$; see the Supplemental Material (SM) [50] for further details on particle preparation and cluster formation]. To create mixtures of oppositely charged titania and silica particles or oppositely charged silica particles, these colloids are dispersed and mixed in tetrahydrofuran (THF, $\varepsilon=7.58$ ).

In a mixture containing positively charged PMMA particles $\left(\sigma_{B}=4025 \mathrm{~nm}\right.$, red, Fig. 1) and negatively charged phosphoric acid 2-hydroxyethyl methacrylate (PAM) PAM-PMMA particles $\left(\sigma_{A}=1380 \mathrm{~nm}\right.$, green, Fig. 1; see Supplemental Material [50] for details about the synthesis), regular $A B_{n}$-type clusters are formed, with $n=1-6$. Dimers [ $n=1$, Fig. 1(a)], linear $[n=2$, Fig. 1(b)], triangular $[n=3$, Fig. 1(c)], and tetrahedral $[n=4$, Fig. 1(d)] clusters are observed in a sample where the number ratio between satellite and core particles is $N_{B}: N_{A}=10: 1$ (the total volume fraction is just below $10 \%)$. This size ratio $\left(\sigma_{B}: \sigma_{A}=3: 1\right)$ ensures that no more than four of the larger particles can fit around a smaller core particle. Three-dimensional tetrahedral clusters are not immediately apparent from the confocal $X Y$ slices, but become visible by combining a selection of slices. A selection of slices from different focal heights are depicted in Fig. 1(d1), 1(d2), and 1(d3). From the entire stack of

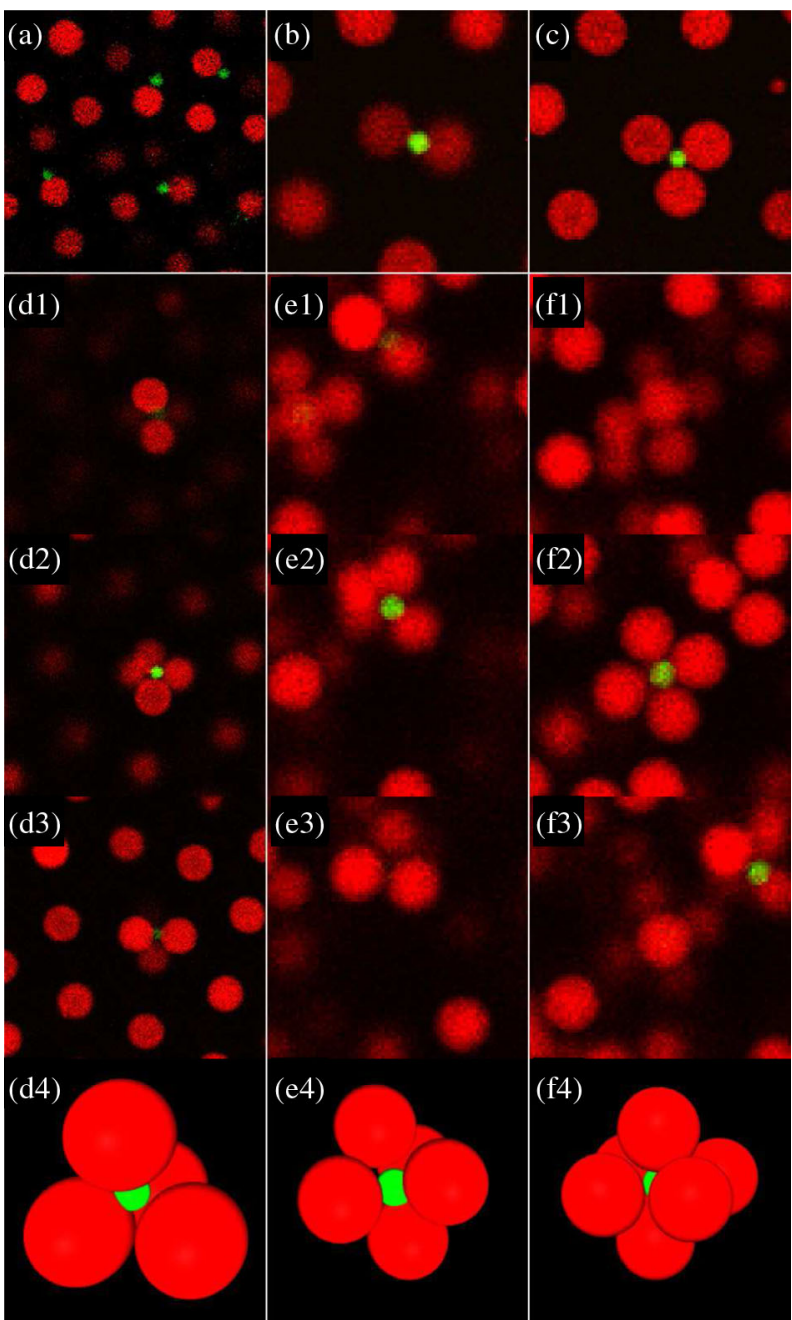

FIG. 1. Confocal microscopy images and computer-generated images of observed clusters consisting of a negatively charged core PMMA particle surrounded by one or more positively charged PMMA particles in $\mathrm{CHB} /$ cis-decalin. In a mixture of positively charged $\left(\sigma_{B}=4025 \mathrm{~nm}\right.$, red) and negatively charged particles $\left(\sigma_{A}=1380 \mathrm{~nm}\right.$, green), $\sigma_{B}: \sigma_{A}=3: 1$, (a) dimers (b) linear clusters, (c) triangular clusters, and (d) tetrahedral clusters are formed. When slightly smaller satellite particles $\left(\sigma_{B}=3025 \mathrm{~nm}\right.$, red) are used $\left(\sigma_{B}: \sigma_{A}=2.2: 1\right)$, (e) triangular bipyramids and (f) octahedral clusters are formed. (d4)-(f4) Computer-generated 3D reconstructions from the particles' coordinates obtained from confocal images at different heights in the sample.

images, particle coordinates are extracted, which are then used to computer generate a three-dimensional model, shown in Fig. 1(d4).

In a different sample (sample 7, see SM for details [50]), where smaller satellite PMMA particles are used $\left(\sigma_{B}=3025 \mathrm{~nm}\right.$, red, Fig. 1), the size ratio is slightly different $\left(\sigma_{B}: \sigma_{A}=2.2: 1\right)$. In this case, triangular bipyramid and octahedron clusters are observed [Figs 1(e) and 1(f)], together with the smaller clusters. Figures $1(\mathrm{e} 1)-1(\mathrm{e} 4)$ and 1(f1)-1(f4) show the three confocal images taken at different 
heights for each cluster together with a three-dimensional rendering of the structure. In our experiments, we observe a clear difference between locked and unlocked PMMA particles. In locked particles, the stabilizer has been covalently bound to the surface of the colloids by an extra reaction step (see SM for details [50]), whereas in unlocked particles, it is physically adsorbed. Locked satellite particles repel each other much more strongly than unlocked particles [51], and as a result, smaller clusters are observed. The clusters ranging from dimers $(A B)$ to tetrahedra $\left(A B_{4}\right)$ are all obtained from locked particles. The particles that form triangular bipyramid and octahedron clusters are unlocked. These observations agree with the simulations (described in more detail below); i.e., an increase in the charge of the satellite particles decreases the number of satellite particles [Fig. 6(a)].

Oppositely charged silica particles dispersed in THF also form regular clusters, which repel each other due to a net charge of the clusters, as shown in Fig. 2(a) (see also Movie $\mathrm{S} 1$ in SM [50]). It is observed that the cluster-cluster distance reaches up to $4 \mu \mathrm{m}$ in THF, whereas the cluster itself has a size of approximately $2 \mu \mathrm{m}$. Here, the estimated Debye screening length $\kappa^{-1}$ is $\sim 1.3 \mu \mathrm{m}$. The clusters are coated with an extra layer of silica to permanently bind the colloids to each other. To fix the clusters, a $50 \mu \mathrm{L}$
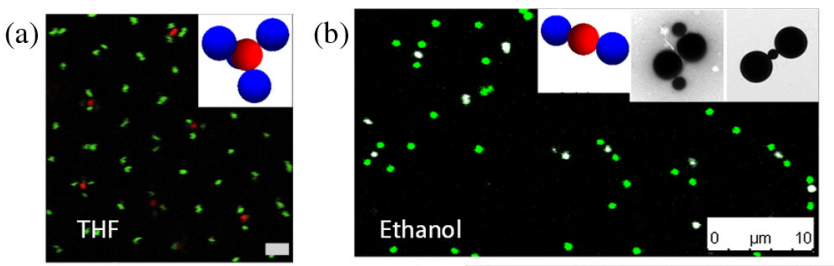

(c)
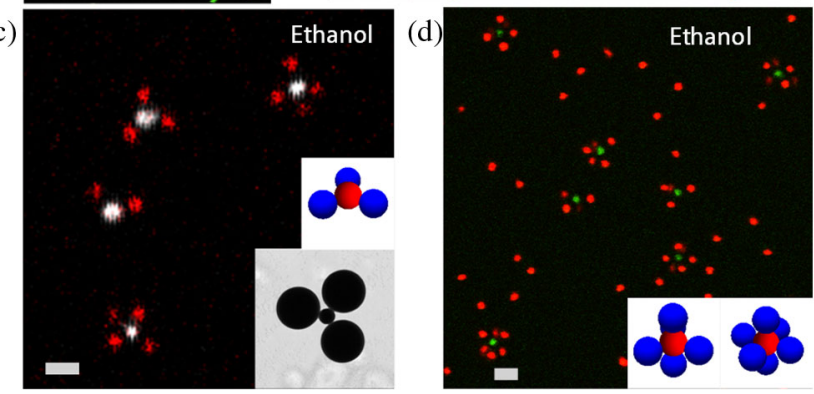

FIG. 2. Clusters composed of positively charged titania and negatively charged silica or of positively and negatively charged silica-silica colloids: (a) $600 \mathrm{~nm}$ positively charged silica (red) and $1.1 \mu \mathrm{m}$ negatively charged silica (green) particles in THF, which still retain the repulsions between the clusters (forming mainly $A B_{3}$ and $A B_{4}$ clusters), (b) $450 \mathrm{~nm}$ positively charged titania (white) particles permanently linked to the $1.7 \mu \mathrm{m}$ negatively charged silica (green) particles in ethanol, which form mainly $A B_{2}$ clusters, (c) $450 \mathrm{~nm}$ positively charged titania (white) particles permanently linked to the $1.3 \mu \mathrm{m}$ negatively charged silica (red) particles in ethanol, (d) $1.1 \mu \mathrm{m}$ positively charged silica (green) permanently linked to $1.3 \mu \mathrm{m}$ negatively charged silica (red) particles in ethanol, which form mainly $A B_{5}$ and $A B_{6}$ clusters. Insets show transmission electron micrographs and schematic drawings of permanently linked clusters. concentrated ammonia solution (28\%-30\% aqueous ammonia solution) is added into a $2 \mathrm{~mL}$ THF solution of particles and is followed by an addition of $20 \mu \mathrm{L}$ tetraethoxysilane. The mixture is rolled for $2 \mathrm{~h}$ on rolling cylinders for the completion of the reaction at a speed of $120 \mathrm{rpm}$. Permanent clusters are dispersed in ethanol to show the stability of the structures [Figs 2(b)-2(d)]. The clusters could also be composed of positively charged titania particles and negatively charged silica particles, after which they could be made permanent. Figure 2(b) shows permanent clusters for $450 \mathrm{~nm}$ positively charged titania surrounded with $1.7 \mu \mathrm{m}$ negatively charged silica particles, yielding predominantly $A B_{2}$ clusters. Figure 2(c) shows $450 \mathrm{~nm}$ positively charged titania particles permanently linked to the $1.3 \mu \mathrm{m}$ minus-charged silica particles, resulting in predominantly $A B_{3}, A B_{4}$ clusters. Finally, Fig. 2(d) shows the $1.1 \mu \mathrm{m}$ positively charged silica and $1.3 \mu \mathrm{m}$ negatively charged silica particles, yielding $A B_{5}$ and $A B_{6}$ clusters (see also Supplemental Movies S2-S4 [50]). TEM images and schematic drawings of these fixed clusters are also given as insets. During the fixation of the clusters with extra silica, especially when the coating is very thin (i.e., 10-30 nm), some satellite particles that are not firmly held detach and result in $A B_{n-1}$ or $A B_{n-2}$ structures, similar to water and ammonia molecules. Figure 2(c) shows one $A B_{2}$ cluster with the same symmetry as water molecules; see also SM Fig. S2 and Supplemental Movies S6 and S7 for more examples of broken clusters.

The yield of clusters for particles with different size ratios shown in Fig. 2(b)-2(d) is summarized in Table II. Clusters with $A B_{2}-A B_{6}$ structures are observed for different size ratios $\sigma_{B}: \sigma_{A}$ of satellite and core particles. We have also observed higher- $n$ clusters, but keep our focus on the smaller clusters; see SM Fig. S2. Simply considering the size ratio of the core and the satellite particles uncovers a trend for the dominant cluster type, as shown in Table I. It is observed that as the size ratio between the satellite particles and the core particles $\sigma_{B}: \sigma_{A}$ increases, the number of satellite particles $n$ decreases. However, simulation results show that the cluster formation depends not only on the size ratio but also on some other factors, such as screening length of the solvent and the charges and densities of both particles.

To study the role of the screening length, we investigate the influence of ionic strength in samples containing clusters of oppositely charged PMMA particles. The ion concentration is increased by adding tetrabutylammonium bromide (TBAB) salt. We use mixtures containing positively charged particles $\left(\sigma_{B}=4025 \mathrm{~nm}\right.$, red, Fig. 1) and locked negatively charged PAM-PMMA particles $\left(\sigma_{A}=1380 \mathrm{~nm}\right.$, green, Fig. 1). The size ratio of $\sigma_{B}: \sigma_{A} \approx$ 3:1 ensures that no more than four positive particles physically fit around a negative particle. An excess amount of larger positive particles is used $\left(N_{B}: N_{A}=10: 1\right)$. Confocal $Z$ stacks containing on the order of 5000 central and satellite particles are recorded in a total particle volume 
TABLE I. Size of core and satellite particles and resulting cluster types for mixtures of positively charged core particles and negatively charged satellite particles. For the core particles, either titania or silica is used. Satellite particles are always made of silica.

\begin{tabular}{lccc}
\hline \hline $\begin{array}{l}\text { Core material } \\
\text { and diameter } \sigma_{A}\end{array}$ & $\begin{array}{c}\text { Satellite material } \\
\text { and diameter } \sigma_{B}\end{array}$ & $\begin{array}{c}\text { Size ratio } \\
\sigma_{B}: \sigma_{A}\end{array}$ & $\begin{array}{c}\text { Main cluster } \\
\text { type }\end{array}$ \\
\hline Titania $450 \mathrm{~nm}$ & Silica $1.7 \mu \mathrm{m}$ & $3.8: 1$ & $A B_{2}$ \\
Titania $450 \mathrm{~nm}$ & Silica $1.3 \mu \mathrm{m}$ & $2.9: 1$ & $A B_{3}, A B_{4}$ \\
Silica $600 \mathrm{~nm}$ & Silica $1.3 \mu \mathrm{m}$ & $2.2: 1$ & $A B_{2}$ \\
Silica $600 \mathrm{~nm}$ & Silica $1.1 \mu \mathrm{m}$ & $1.8: 1$ & $A B_{3}, A B_{4}$ \\
Silica $1.1 \mu \mathrm{m}$ & Silica $1.3 \mu \mathrm{m}$ & $1.2: 1$ & $A B_{5}, A B_{6}$ \\
\hline \hline
\end{tabular}

fraction of $10 \%$. We estimate the Debye screening length $\kappa^{-1}$ to be $\sim 2 \mu \mathrm{m}$ for this system before adding any salt. The TBAB concentration in the suspension is increased from 0 to $\sim 2 \mu \mathrm{M}$ by adding appropriate amounts of diluted TBAB in CHB. The Debye screening lengths $\kappa^{-1}$ for TBAB concentrations of $0 ; 0.5 ; 1 ; 1.5 ; 2 \mu \mathrm{M}$ are estimated to be $2.0 ; 1.3 ; 1.0 ; 0.9 ; 0.8 \mu \mathrm{m}$. From these $Z$ stacks, coordinates of the particles and different cluster compositions are identified. The results of these analyses show that in all samples the cluster composition peaks at a certain configuration. This peak is found to slightly shift from $n=3$ (triangular) to $n=2$ (linear) with increasing TBAB concentration in our samples, until clusters fall apart when the TBAB concentration reaches approximately $2 \mu \mathrm{M}$ (see Fig. S1 in SM [50]). One might expect that a smaller double layer would allow more satellite particles to fit around a core particle. However, a reduction in Debye screening length also reduces the attraction between the central and satellite particles and causes fewer satellite particles to bind to a core particle, while on the other hand, the screening becomes so high above a certain threshold concentration of ions that the behavior of the particles is no longer governed by electrostatic repulsions or attractions (see Fig. 3). Occasionally, some clusters with two or three central particles $\left(A_{m} B_{n}\right)$ are also found (Fig. S1 in SM [50]). However, the formation of these more complex clusters is largely suppressed by the large excess of satellite particles, making it unlikely for a central particle to stick to a satellite that is already attached to another core.

Finally, we mention here that the clusters of oppositely charged colloids presented here are not the equilibrium

TABLE II. Yields of the titania-silica or silica-silica clusters for different size ratios. The cluster type is given as the type of core $(A) /$ satellite $(B)$ particles and their respective size ratios, $\sigma_{B}: \sigma_{A}$. The percentages were estimated by analyzing $100-150$ clusters, ignoring excess satellite particles that were not part of a cluster.

\begin{tabular}{lcccccc}
\hline \hline$A / B\left(\sigma_{B}: \sigma_{A}\right)$ & $A B$ & $A B_{2}$ & $A B_{3}$ & $A B_{4}$ & $A B_{5}$ & $A B_{6}$ \\
\hline $\mathrm{TiO}_{2} / \mathrm{SiO}_{2}(3.8: 1)$ & $44 \%$ & $54 \%$ & $2 \%$ & & & \\
$\mathrm{TiO}_{2} / \mathrm{SiO}_{2}(2.9: 1)$ & & $27 \%$ & $38 \%$ & $35 \%$ & & \\
$\mathrm{SiO}_{2} / \mathrm{SiO}_{2}(1.2: 1)$ & & & $7 \%$ & $19 \%$ & $35 \%$ & $39 \%$ \\
\hline \hline
\end{tabular}
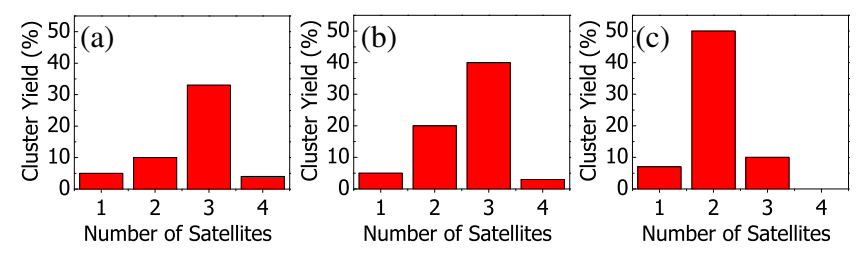

FIG. 3. Normalized incidence of clusters found in a suspension containing positively charged PMMA particles $\left(\sigma_{B}=4025 \mathrm{~nm}\right)$ and locked negatively charged PAM-PMMA particles $\left(\sigma_{A}=1380 \mathrm{~nm}\right)$, (a) without added salt, (b) with $0.5 \mu \mathrm{M}$ TBAB, and (c) with $1.0 \mu \mathrm{M}$ TBAB concentration. The complete data including yield of clusters with more than one central particle (typically less than 5\%-10\%) are shown in Fig. S1 of SM [50]. Note also that the yield for a certain cluster type is given here by the fraction of the number of observed clusters to the maximum number of that cluster type that could have formed given the total number of central particles.

structures; instead, the clusters are metastable with respect to larger aggregates of oppositely charged particles. However, the long-range repulsion between the clusters that have similar charge to the satellite particles kinetically traps them at a final cluster size, which provides enough charge to repel the other particles. Provided that the satellite particles are in sufficient excess to saturate all core particles, the metastable clusters can be reproducibly fabricated. When the satellite:core number ratio $N_{B}: N_{A}$ is decreased to $2: 1$, parts of the core particles remain unshielded, and the clusters can grow in size and form ring or chainlike structures (see Fig. S3 in SM [50]).

\section{B. Tuning the particle interactions with electric fields}

To examine how firmly satellite particles are attached to oppositely charged cores in the case of the sterically stabilized PMMA spheres, we expose samples to an electric field. Insights in this behavior are also relevant for the mechanism of equilibrium phases formed between these PMMA model particles as it was hypothesized and expected that the particles had to be able to still move over each other's surface after becoming attached by the screened Coulomb interactions [17]. For this experiment, we first create raspberrylike regular clusters of oppositely charged particles by mixing large polymethylmethacrylate (PMMA) particles $\left(\sigma_{L}=3.68 \mu \mathrm{m}\right)$ with a surplus of small PMMA particles $\left(\sigma_{S}=0.64 \mu \mathrm{m}\right)$ [52], such that the number ratio $N_{S}: N_{L} \approx 388: 1$ at a total volume fraction of $\phi=0.02$. Then the mixture is transferred into a glass capillary with two conductive wires threaded along on either side (see Ref. [53] for details on the experimental setup) and studied with laser scanning confocal microscopy. In Fig. 4, it can be seen that a large amount of positively charged satellite particles adsorb on the surface of the large negatively charged particle, in a way similar to that described in Ref. [34], and with a uniform distribution. From 3D confocal microscopy data sets, we determine that there are typically between 50 and 70 small satellite 

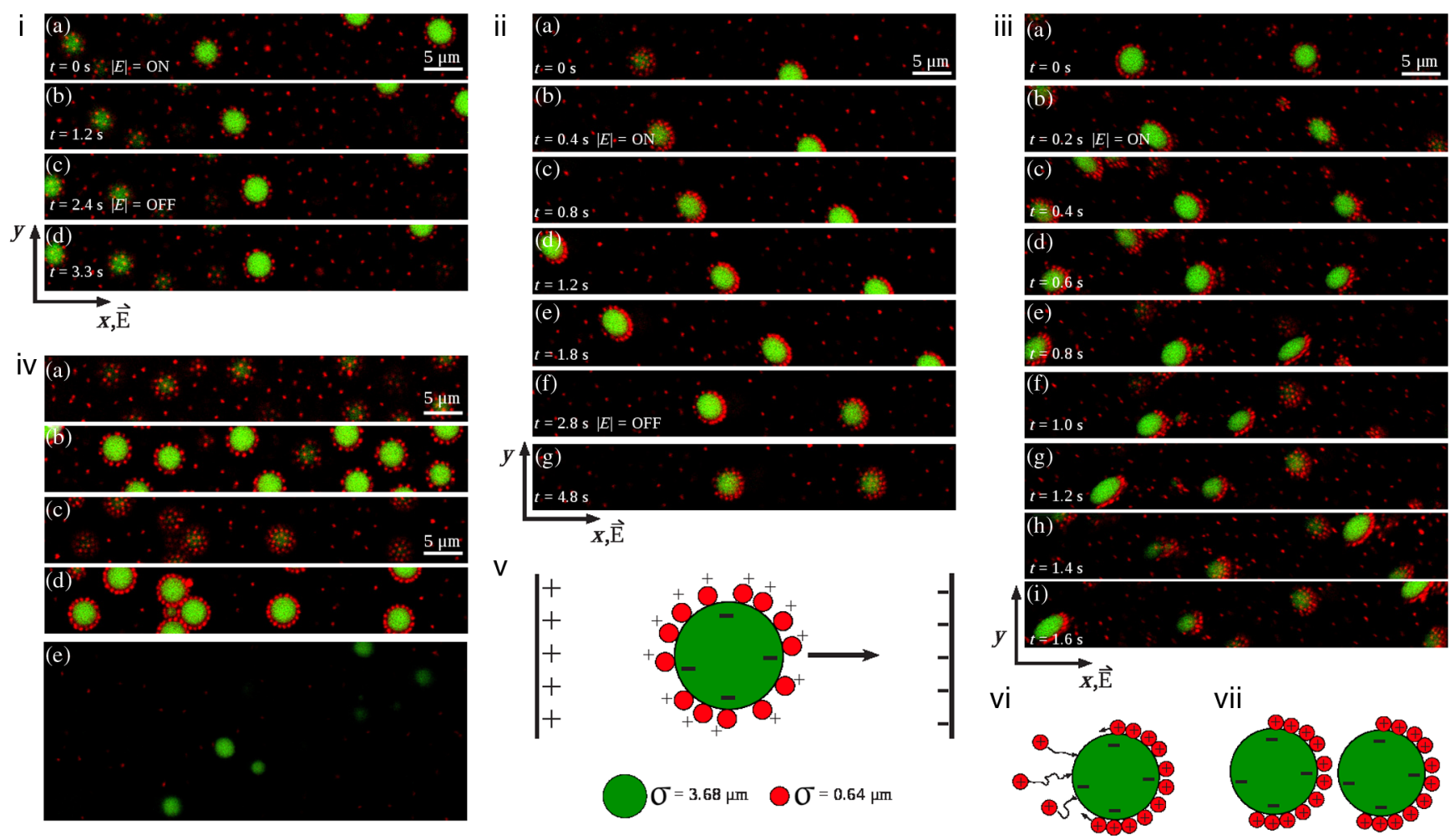

FIG. 4. Electrophoresis experiments of clusters made of large negatively charged $\left(\sigma_{L}=3.68 \mu\right.$ m $)$ and small positively charged colloidal particles $\left(\sigma_{S}=0.64 \mu \mathrm{m}\right)$. (ia)-(id) Time series of confocal images recorded for a field strength $|E|=11 \mathrm{~V} / \mathrm{mm}$. The clusters move towards the negative electrode, indicating that the net charge on the clusters is positive. (iia)-(iig) Time series of images recorded for $|E|=29 \mathrm{~V} / \mathrm{mm}$. The small positively charged particles "crawl" over the surface to one pole of the large, negatively charged particle. Note that the large particles appear deformed due to their fast movement compared to the line scanning speed of the microscope. (f) After the field is turned off, the particles redistribute again isotropically over the surface. Before this process is completed, the bare side of the large particle remains more vulnerable to the attachment of other, additional positively charged small particles that were previously in solution. (iiia)-(iiii) Images from a time series recorded at $|E|=77 \mathrm{~V} / \mathrm{mm}$. In the first image (a), no field is applied. During the first $\sim 0.6 \mathrm{~s}$ in which the field is applied (b)-(d), clusters move towards the negative electrode. Small particles (red) accumulating on one side of the large particle (green) cause an anisotropic charge distribution on the cluster. At some point between 0.4 and $0.6 \mathrm{~s}$ after the field is turned on (d),(e), the clusters as a whole reverse direction (e)-(i), indicating that a sufficient number of small particles has been pulled from the large particle by the electric field to reverse the net charge of the cluster. (iva)-(ivd) Confocal microscopy images of clusters before (a),(b) and after (c),(d) an electric field $(|E|=29 \mathrm{~V} / \mathrm{mm}$ ) is applied in 9 cycles of $2.5 \mathrm{~s}$ each. In between these cycles, the field is switched off for $7.5 \mathrm{~s}$. (e) After an electric field $(|E|=77 \mathrm{~V} / \mathrm{mm}$ ) is applied in 9 cycles of $2.5 \mathrm{~s}$ each (the field is switched off for $7.5 \mathrm{~s}$ between each cycle), bare large negatively charged particles are found near the positive electrode, indicating that (almost) all of the small particles have been pulled off. Schematic representations (not drawn to scale) of (v) an unpolarized, net positively charged cluster moving in an electric field, (vi) the adsorption of extra small particles on a polarized cluster, and (vii) the attachment of one polarized cluster to another one.

particles on the surface of a large particle. The clusters as a whole mutually repel each other over distances of several micrometers; see Supplemental Movie S8 [50], showing that the electrostatic interactions are quite long ranged. From the measured conductivity [54] of the solvent $(\sigma=70 \mathrm{pS} / \mathrm{cm})$, we estimate the screening length to be $\kappa^{-1}=1.82 \mu \mathrm{m}$.

In previous papers on mixtures of oppositely charged particles in electric fields, the collective dynamics were investigated for two particle species having approximately the same size $[53,55]$. Here, we show that it is also possible to use electric fields to change the properties and interactions of individual building blocks, in this case, clusters of oppositely charged large and small particles. To study the dynamics and interactions of the clusters, we perform electrophoresis experiments in which both the clusters and unbound positively charged particles are driven by a dc electric field. In each experiment, we run a number of cycles in which we apply an electric field for $2.5 \mathrm{~s}$, after which we switch the field off for $7.5 \mathrm{~s}$. For each cycle, a series of confocal images is recorded to study the evolution of the clusters while they are moving under the influence of the electric field. Figures 4(i)-4(iii) display experiments performed at different field strengths for clusters of large particles with diameter $\sigma=3.68 \mu \mathrm{m}$ and small particles $\sigma=0.64 \mu \mathrm{m}$. 
Figures 4(ia)-4(id) show a time series of confocal images recorded at a field strength of $|E|=11 \mathrm{~V} / \mathrm{mm}$. A movie of the sequence is also given as Supplemental Movie S9 [50]. The clusters move towards the negative electrode, indicating that the net charge on the clusters has the same positive sign of charge as the satellite particles. This so-called "overcharging" of the large particle by the small satellite particles is consistent with results that were found for clusters of oppositely charged particles that interacted via a Coulomb potential [56], and with earlier work by Vincent et al. [34].

From the confocal microscopy images, we extract electrophoretic mobilities of the small particles $\mu_{S}=$ $200-300 \mu \mathrm{m}^{2} / \mathrm{V} \mathrm{s}$, from which the charge on the small particles is estimated [53,54] between $Z_{S} e=+25 e$ and $Z_{S} e=+100 e$, where $e$ is the absolute electron charge. From the electrophoretic mobilities of the clusters, in the range $\mu_{\mathrm{tot}}=200-400 \mu \mathrm{m}^{2} / \mathrm{V} \mathrm{s}$, we obtain approximate charges for the cluster as a whole $Z_{\mathrm{tot}} e$ between $+200 e$ and $+1000 e$. From this considerable spread, we estimate the charge on the large particles $Z_{L} e=Z_{\text {tot }} e-n_{S} Z_{S} e=$ $-3150 e \pm 2650 e$. More details can be found in the SM [50].

In a follow-up experiment, a stronger field of $|E|=$ $29 \mathrm{~V} / \mathrm{mm}$ is applied. The result is shown in Fig. 4(ii) and Supplemental Movie S10 [50]. Surprisingly, the positively charged small particles start "crawling" over the surface of the larger negatively charged particles, resulting in an anisotropic particle distribution on the surface of the large particles. When the field is turned off, the structure relaxes and the particles redistribute uniformly over the surface. Before the redistribution is complete, however, the underpopulated side of the large particle collects additional small satellite particles from the suspension. From 3D data sets, we indeed find that after several cycles where the electric field is applied, clusters typically contain 70-80 satellite particles instead of the initial 50-70 particles. In addition to the attachment of extra small particles, we observe that polarized clusters could also attach to the unprotected side of another polarized cluster when the field is turned on. Because not all of the clusters move exactly at the same speed, collisions occur once in a while, further enhancing this process. Figures 4(iva)-4(ivd) illustrate both the merging of clusters and the increase of the number of satellite particles as a result of the electric field cycles. Figures. 4(iva) and 4(ivb) show the clusters before the field is applied, and Figs. 4(ivc) and 4(ivd) show them after an electric field $(|E|=29 \mathrm{~V} / \mathrm{mm})$ has been applied for 9 cycles of $2.5 \mathrm{~s}$ each. In between these cycles, the field is switched off for $7.5 \mathrm{~s}$.

For even stronger electric fields, small satellite particles could be stripped off completely from the host particle. Figure 4(iii) and Supplemental Movie S11 [50] show the sequence of events when a field of $|E|=77 \mathrm{~V} / \mathrm{mm}$ is applied to dispersion. Initially, the clusters start moving towards the negative electrode, while they become strongly polarized as the positively charged satellite particles move over the surface towards one pole. Eventually, some of the small particles are pulled off from the surface. After $\sim 0.5 \mathrm{~s}$, the clusters change direction and start moving to the positively charged electrode, indicating that their net charge must have been reversed. Figure 4(ive) shows that after the field has been applied for a long time, "bare" negatively charged particles are present close to the positive electrode, showing that (almost) all of their satellite particles have been stripped off. A schematic representation of net negatively charged, unpolarized cluster in an electric field is shown in Fig. 4(v). Schematic representations of the adsorption of extra small particles to a polarized cluster, and of how two polarized clusters can easily attach to each other, are depicted in Figs. 4(vi) and Fig. 4(vii), respectively.

\section{Simulations}

We use Monte Carlo simulations to study clusters of oppositely charged colloidal particles interacting via a screened Coulomb potential, where multiple particles of one species are clustering around a single particle with an opposite charge. The pair interaction potential we use is given by a hard-core repulsion $U_{\mathrm{HS}}$, plus a screened Coulomb potential $U(r)$, using the linear superposition approximation [57]:

$$
\beta U(r)=\beta U_{\mathrm{HS}}(r)+\frac{Z_{i} Z_{j}}{\left(1+\kappa \sigma_{i} / 2\right)\left(1+\kappa \sigma_{j} / 2\right)} \lambda_{B} \frac{\exp (\kappa r)}{r},
$$

where $r$ is the distance between two particles, $\sigma_{i}$ is the diameter of particle $i, \kappa$ is the inverse Debye screening length, $\lambda_{B}$ is the Bjerrum length in the solvent, and $Z_{i}$ is the charge (measured in electron charges) on particle $i$. We note here that this simple potential is likely not quantitatively accurate for the colloids used in the experiments. In particular, the approximation we use here that van der Waals forces are not important is not expected to be valid at the short interparticle distances involved in some of the cluster formation. Furthermore, the double-layer overlap potential we use ignores the possibility of charge renormalization, any dependence of the particle charge or screening length on the density of the colloids, and other many-body effects. However, as our simulations show, this simple model is sufficient to qualitatively capture the behavior of the experimental system.

For a given number of satellite charges, the location of the satellite particles has a well-defined energy minimum due to the repulsions between the (identically charged) satellites. In the case of an unscreened Coulomb potential $(\kappa=0)$, this optimization problem is known as the 
Thomson problem, and solutions are known for a large range of cluster sizes [47,58]. However, proving that a solution actually possesses the lowest energy is not trivial [47], and for many larger cluster sizes, this has not yet been done. To determine the optimal locations of the satellite particles interacting via screened Coulomb potentials, we simulate single clusters with a fixed number $n$ of satellite particles constrained to be in contact with the core particle, where the interaction strength (charge) of the particles is slowly increased until the particles no longer significantly move. The final configurations from multiple independent runs are compared with each other to find the global energy minimum, which is then compared with the known solutions to the Thomson problem.

We investigate the energy minima for inverse Debye screening lengths $0 \leq \kappa \sigma \leq 10$ and a size ratio of 1 (i.e., the satellites and core particle have the same hard-core diameter $\sigma$ ), which means that up to $n=12$ particles can touch the core sphere without overlapping. It should be noted that the size ratio only affects the ground state when the satellite particles are large enough to touch each other. In practice, this occurs only for cluster size $n=11$. For $n \leq 6$, we observe that the ground-state structures formed exactly coincide with the solutions to the Thomson problem (see Fig. 5). At $n=7$, a different structure has a lower energy at $\kappa \sigma>2$, consisting of an equilateral triangle of particles around one pole, a wider triangle rotated by $60^{\circ}$ parallel to it close to the equator, and one particle at the other pole. For $n=8$ and $n=9$, the same symmetries as in the Thomson problem appear again, but particles shift along the symmetry axis as $\kappa$ changes: for instance, at $n=8$, the Thomson solution is a square antiprism (or anticube). For a screened potential, the solution is still a square antiprism, but the distance between the two parallel planes of the prism changes as a function of $\kappa$. For $n=10$, the structure for $\kappa \sigma>7$ is similar to the one at $n=7$ : in this case, there are three parallel equilateral triangles, and again one particle at a pole (see bottom right of Fig. 5). For $n=11$, the ground state for the Thomson problem causes overlap in our Yukawa particles. For low $\kappa$, the ground state in the Yukawa case is a slight variation on this cluster, which has particles touching, and lacks any noticeable symmetry. For $\kappa \sigma>3$, the energy minimum shifts to a different configuration, again lacking significant symmetry. At $n=12$, the icosahedron is the lowest-energy state for all investigated values of $\kappa$. In short, for $n=7, n=10$, and $n=11$, inherently different structures from the solutions to the Thomson problem are found for $\kappa \sigma>2, \kappa \sigma>7$, and $\kappa \sigma>3$, respectively.

The structures of the clusters formed by oppositely charged particles are qualitatively different from those obtained by emulsion droplet evaporation [10,59], in that they always contain a core particle at the center. In contrast, clusters formed via emulsion droplet evaporation rarely show this feature. Nonetheless, if we consider only the (a) $n$
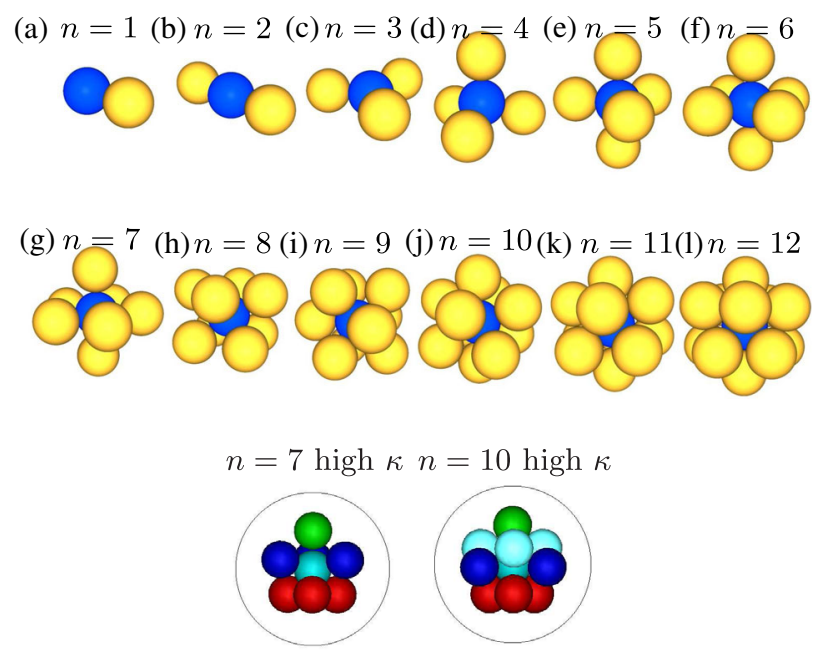

FIG. 5. Optimal configurations for $A B_{n}$ clusters. Negatively charged blue particle and positively charged $n$ ( $n \epsilon$ [1,12]) yellow particles are the same size: (a) dimer. (b) line, (c) triangle, (d) tetrahedron, (e) triangular bipyramid, (f) octahedron, (g) pentagonal bipyramid, (h) anticube, (i) triaugmented triangular prism, (j) gyroelongated square bipyramid, (k) irregular, (l) icosahedron. The bottom row shows the alternative clusters found only for short screening lengths (high $\kappa$ ). (Left) Lowestenergy cluster of 7 particles. A threefold rotational axis of symmetry goes through the (light blue) core particle and the green particle. The other two colors show different equilateral triangles perpendicular to the plane of view. (Right) Lowestenergy cluster of 10 particles.

satellite particles, then the minimum-energy clusters for low $\kappa$ are essentially the same as those produced using emulsions. The main differences are a distortion of the gyroelongated square dipyramid for $n=10$, which renders it more spherical, and a different irregular configuration for $n=11$. Interestingly, the high- $\kappa$ structure for $n=7$ is also observed in emulsion droplet evaporation experiments [59], unlike the one for $n=10$.

At finite temperatures, the particles in the cluster fluctuate around their ideal positions, and the number of satellite particles in the cluster can vary, as particles can escape and join the cluster from the solution around it. To illustrate the requirements for obtaining clusters of specific sizes, we perform NVT Monte Carlo simulations of $N_{c}=50$ core particles and $N_{s}=500$ satellite particles and measure the average number of satellite particles around each core particle, varying the charge ratio and screening length. Clusters containing more than one core particle are ignored (the few-core-particle clusters are minimal; see Fig S1 of SM for experimental results [50]). Note that it is possible that, in some of these simulations, the formation of clusters is metastable with respect to phase separation or crystallization. However, in our simulation, we do not see signs of this. However, for small values of $Z_{s} / Z_{c}$, the simulated systems sometimes phase separate into a denser phase rich in clusters and a more dilute phase consisting 

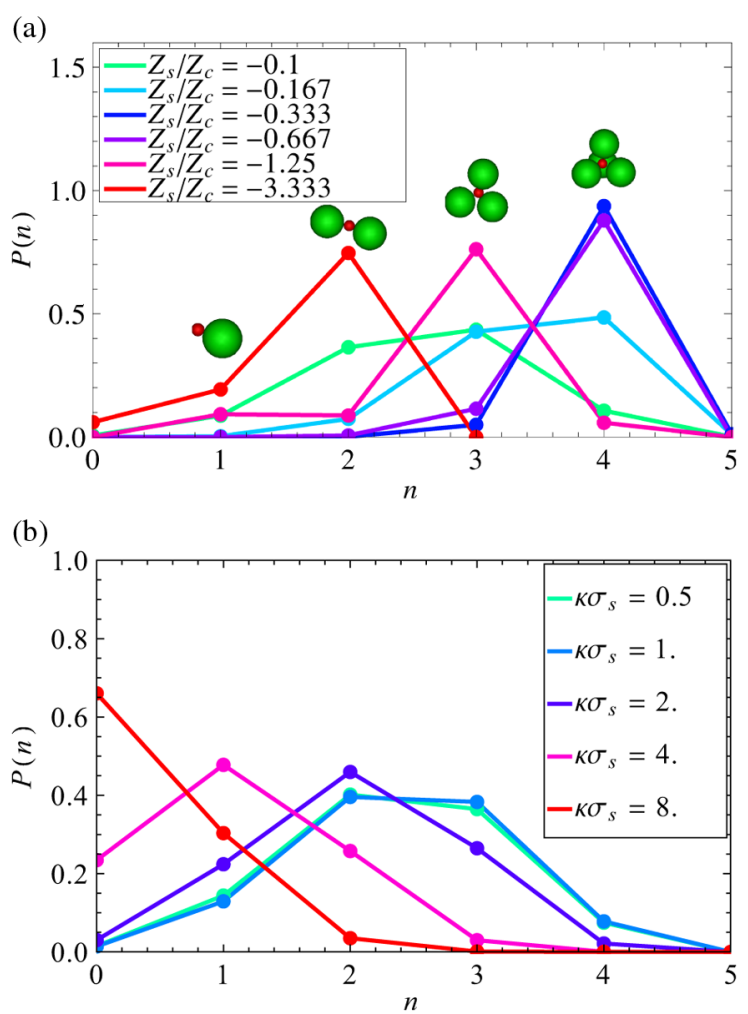

FIG. 6. Probability distributions of finding $n$ satellite particles around a core particle, for size ratio $\sigma_{s} / \sigma_{c}=3 / 1$. (a) Distributions for different values of the charge ratio between satellite and core particles $Z_{s} / Z_{c}$, at a constant Debye screening length $\kappa \sigma_{s}=1$, where $\sigma_{s}$ is the diameter of a satellite particle. (b) Distributions for different values of the inverse Debye screening length $\kappa \sigma_{s}$, at a constant charge ratio $Z_{s} / Z_{c}=-0.125$. Clusters that contain more than one core particle are not included in the calculation. In all cases, the charge of the core particles is kept constant, such that $Z_{c}^{2} \lambda_{B} / \sigma_{s}=108.8$, the number ratio $N_{s} / N_{c}=10$, and the total packing fraction $\eta=0.10$.

almost purely of satellite particles. Figure 6 shows the probability of finding $n$ satellite particles around a core particle for size ratio $\sigma_{s} / \sigma_{c}=3$, at constant core particle charge, but varying satellite particle charge (top) and Debye screening length (bottom). For this size ratio, the largest clusters observed contain four satellite particles, arranged in a tetrahedral geometry, analogous to the clusters seen in the experiments. Interestingly, increasing the charge of the satellite particles beyond a certain optimum decreases the number of particles in the cluster, analogous to what we observe when using locked particles in the experiments. Additionally, similar to the experimental results shown in Fig. 3, decreasing the Debye screening length (increasing $\kappa)$ reduces the number of particles in the cluster as well, due to the decreased attraction between core and shell particles. Figure 7 shows cluster size distributions, for size ratio $\sigma_{s} / \sigma_{c}=2.2$. In this case, we observe the formation of clusters with up to six satellite particles, in an octahedral geometry similar to those seen in the experiments using the

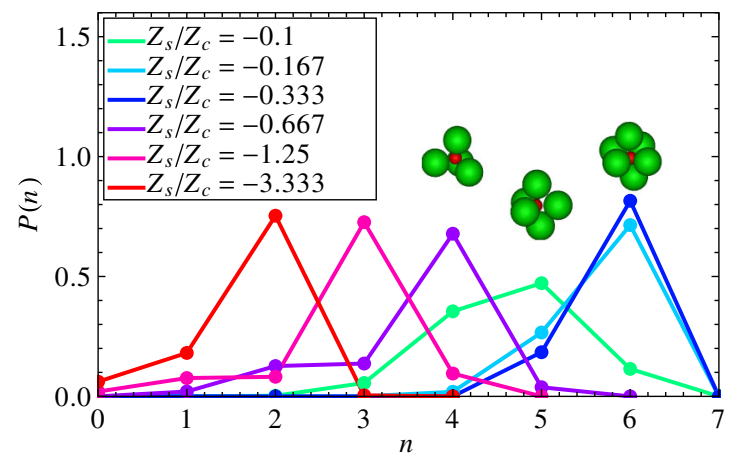

FIG. 7. Probability distribution of finding $n$ satellite particles around a core particle, for size ratio $\sigma_{s} / \sigma_{c}=2.2 / 1$, and for different values of the charge ratio $Z_{s} / Z_{c}$. Here, the Debye screening length is fixed at $\kappa \sigma_{s}=1$, the core charge $Z_{c}^{2} \lambda_{B} / \sigma_{s}=108.8$, the number ratio $N_{s} / N_{c}=10$, and the total packing fraction $\eta=0.10$.

same size ratio. Again, we see that the number of satellite particles in the cluster increases with increasing satellite charge until a maximum is reached. Upon increasing the satellite charge further, the number of satellite particles per cluster decreases again. While quantitatively comparing distributions with the experimental system is not possible due to the fact that the experimental particle charge, screening length, and Bjerrum length are not accurately known, these trends agree well with those seen in the experiments.

\section{CONCLUSIONS}

In summary, we demonstrate a facile route for fabrication of well-defined binary colloidal clusters, based on concurrent attractive and repulsive Yukawa interactions. The clusters, another example of "complex colloids" [60], form as a result of self-assembly of the oppositely charged particles, resulting in binary colloids that are mesoscopic analogs of binary molecules and other regular clusters without molecular equivalents. We observe a class of particles with a symmetry of molecules, like $\mathrm{HCl}$ (dimer, $A B$ ), $\mathrm{BeCl}_{2}$ (linear, $A B_{2}$ ), $\mathrm{BF}_{3}$ (triangular, $A B_{3}$ ), $\mathrm{CH}_{4}$ (tetrahedron, $A B_{4}$ ), $\mathrm{PCl}_{5}$ (triangular dipyramid, $A B_{5}$ ), and $\mathrm{XeF}_{6}$ (octahedron, $A B_{6}$ ). The repulsions between the surrounding particles also resemble the valence shell electron pair repulsion model, which allows us to predict the molecular geometry. In this procedure, one interesting advantage is that, during the coating step for permanently bonding particles, colloids that mimic molecules with incomplete structures such as bent geometries $\left(\mathrm{H}_{2} \mathrm{O}\right)$ or trigonal pyramids $\left(\mathrm{NH}_{3}\right)$ are also produced, as a result of some satellite particles detaching from the core particle.

The relatively long-ranged screening length we use in the self-assembly compared to the particle size allows regular clusters to be formed without satellite particle-satellite particle contacts on the oppositely charged core particle, 
allowing for cluster geometries not possible with purely repulsive or short-ranged potentials. One other unique property of our method is that it not only provides directionality in bonding similar to the previously described methods [2,11] but also provides tunability in the density of the packing by changing the long-ranged particle interactions (mostly repulsions of the satellite particles). In our case, this enables us to put, e.g., four satellite particles around a large or small central particle of a preferred size, thus tuning the surface coverage and patch size of the final cluster. In addition, because equilibrium crystallization of oppositely charged nanoparticles has already been demonstrated both in organic solvents [15] and in water [46,61], we expect the methods we develop in this paper to also be extendable to these systems.

We present an example of clusters consisting of a large, sterically stabilized PMMA particle surrounded by many smaller positively charged colloids, for which the specific interactions could be changed with an electric field. For low field strengths, the clusters are overcharged by positively charged satellite particles and move towards the negative electrode. For a sufficiently strong external electric field, the satellite particles start "crawling" over the surface towards one pole of the large particle, thus polarizing the clusters. Moving polarized clusters regularly collide in a head-to-tail fashion to form larger aggregates or collect additional small satellite particles on their underpopulated pole during relaxation after the field is turned off. For the strongest electric fields we report here, the satellite particles could be completely pulled off the surface of the central colloid, inverting the total net charge of the cluster.

The novel methods to create and alter individual colloidal building blocks of oppositely charged particles we demonstrate in this paper may find use in self-assembly schemes that exploit long-ranged repulsive interactions $[62,63]$ or the interplay of directional attractive and repulsive interactions on heterogeneously charged particles [64]. Moreover, the clusters created using this method can be used for subsequent self-assembly steps, leading to new structures based on the unique shape of the clusters. These structures could, in principle, be further tuned by modifying the properties of the particles used to create the clusters. For instance, using magnetic colloids as the satellite particles can lead to completely novel structures with interesting properties [65]. In future work, it will be interesting to see if the clusters of the PMMA particles can be made permanent using a simple heating step as was also already demonstrated in Ref. [66]. In addition, recently, a new separation method for clusters of larger sizes has been developed, making it possible to separate the mixtures presented here [67].

\section{ACKNOWLEDGMENTS}

The authors acknowledge funding from EU Nanodirect FP7-NMP-2007-SMALL-1. Parts of this research were performed under Project No. 700.54.321 (T. V.), which was financially supported by the Netherlands Organisation for Scientific Research, Chemische Wetenschappen (NWO/ CW). A. F. D. acknowledges the Swiss National Science Foundation (Ambizione Grant, No. PZ00P2_148040) for financial support, and T. V. acknowledges funding through Marie Curie fellowship 623364 under the FP7-PEOPLE2013-IEF program. F. S and M. D. acknowledge financial support from a NWO-VICI grant.

A. F. D., J.C.P.S., and T. V. contributed equally to this work.

[1] Q. Chen, S. C. Bae, and S. Granick, Directed Self-Assembly of a Colloidal Kagome Lattice, Nature (London) 469, 381 (2011).

[2] Y. Wang, Y. Wang, D. R. Breed, V. N. Manoharan, L. Feng, A. D. Hollingsworth, M. Weck, and D. J. Pine, Colloids with Valence and Specific Directional Bonding, Nature (London) 491, 51 (2012).

[3] M. E. Leunissen, R. Dreyfus, F. C. Cheong, D. G. Grier, R. Sha, N. C. Seeman, and P. M. Chaikin, Switchable Self-Protected Attractions in DNA-Functionalized Colloids, Nat. Mater. 8, 590 (2009).

[4] S. Sacanna, W. T. M. Irvine, P. M. Chaikin, and D. J. Pine, Lock and Key Colloids, Nature (London) 464, 575 (2010).

[5] A. F. Demirörs, P. M. Johnson, C. M. van Kats, A. van Blaaderen, and A. Imhof, Directed Self-Assembly of Colloidal Dumbbells with an Electric Field, Langmuir 26, 14466 (2010).

[6] S. C. Glotzer and M. J. Solomon, Anisotropy of Building Blocks and Their Assembly into Complex Structures, Nat. Mater. 6, 557 (2007).

[7] N. B. Schade, M. C. Holmes-Cerfon, E. R. Chen, D. Aronzon, J. W. Collins, J. A. Fan, F. Capasso, and V. N. Manoharan, Tetrahedral Colloidal Clusters from Random Parking of Bidisperse Spheres, Phys. Rev. Lett. 110, 148303 (2013).

[8] K. M. Ho, C. T. Chan, and C. M. Soukoulis, Existence of a Photonic Gap in Periodic Dielectric Structures, Phys. Rev. Lett. 65, 3152 (1990).

[9] A. van Blaaderen, Colloidal Molecules and Beyond, Science 301, 470 (2003).

[10] V. N. Manoharan, M. T. Elsesser, and D. J. Pine, Dense Packing and Symmetry in Small Clusters of Microspheres, Science 301, 483 (2003).

[11] A. Désert, C. Hubert, Z. Fu, L. Moulet, J. Majimel, P. Barboteau, A. Thill, M. Lansalot, E. Bourgeat-Lami, E. Duguet, and S. Ravaine, Synthesis and Site-Specific Functionalization of Tetravalent, Hexavalent, and Dodecavalent Silica Particles, Angew. Chem., Int. Ed. Engl. 52, 11068 (2013).

[12] H. R. Sheu, M. S. El-Aasser, and J. W. Vanderhoff, Phase Separation in Polystyrene Latex Interpenetrating Polymer Networks, J. Polym. Sci. A 28, 629 (1990).

[13] K. Miszta, J. de Graaf, G. Bertoni, D. Dorfs, R. Brescia, S. Marras, L. Ceseracciu, R. Cingolani, R. van Roij, M. Dijkstra, and L. Manna, Hierarchical Self-Assembly 
of Suspended Branched Colloidal Nanocrystals into Superlattice Structures, Nat. Mater. 10, 872 (2011).

[14] F. X. Redl, K. S. Cho, C. B. Murray, and S. O'Brien, ThreeDimensional Binary Superlattices of Magnetic Nanocrystals and Semiconductor Quantum Dots, Nature (London) 423, 968 (2003).

[15] E. V. Shevchenko, D. V. Talapin, N. A. Kotov, S. O'Brien, and C. B. Murray, Structural Diversity in Binary Nanoparticle Superlattices, Nature (London) 439, 55 (2006).

[16] G. Meng, N. Arkus, M. P. Brenner, and V. N. Manoharan, The Free-Energy Landscape of Clusters of Attractive Hard Spheres, Science 327, 560 (2010).

[17] M. E. Leunissen, C. G. Christova, A. P. Hynninen, C. P. Royall, A. I. Campbell, A. Imhof, M. Dijkstra, R. van Roij, and A. van Blaaderen, Ionic colloidal Crystals of Oppositely Charged Particles, Nature (London) 437, 235 (2005).

[18] A. P. Hynninen, M. E. Leunissen, A. van Blaaderen, and M. Dijkstra, CuAu Structure in the Restricted Primitive Model and Oppositely Charged Colloids, Phys. Rev. Lett. 96, 018303 (2006).

[19] E. Lauga and M. P. Brenner, Evaporation-Driven Assembly of Colloidal Particles, Phys. Rev. Lett. 93, 238301 (2004).

[20] B. Peng, H. R. Vutukuri, A. van Blaaderen, and A. Imhof, Synthesis of Fluorescent Monodisperse Non-Spherical Dumbbell-like Model Colloids, J. Mater. Chem. 22, 21893 (2012).

[21] B. Peng, A. van Blaaderen, and A. Imhof, Direct Observation of the Formation of Liquid Protrusions on Polymer Colloids and Their Coalescence, ACS Appl. Mater. Interfaces 5, 4277 (2013).

[22] B. Peng, F. Smallenburg, A. Imhof, M. Dijkstra, and A. vanBlaaderen, Colloidal Clusters by Using Emulsions and Dumbbell-Shaped Particles: Experiments and Simulations, Angew. Chem., Int. Ed. Engl. 52, 6709 (2013).

[23] D. J. Kraft, W. S. Vlug, C. M. van Kats, A. van Blaaderen, A. Imhof, and W. K. Kegel, Self-Assembly of Colloids with Liquid Protrusions, J. Am. Chem. Soc. 131, 1182 (2009).

[24] Q. Chen, S. C. Bae, and S. Granick, Staged Self-Assembly of Colloidal Metastructures, J. Am. Chem. Soc. 134, 11080 (2012).

[25] T. H. Besseling, M. Hermes, A. Kuijk, B. de Nijs, T. S. Deng, M. Dijkstra, A. Imhof, and A. van Blaaderen, Determination of the Positions and Orientations of Concentrated Rod-like Colloids from 3D Microscopy Data, arXiv: 1406.4985.

[26] J. J. Crassous, P. E. Millard, A. M. Mihut, A. Wittemann, M. Drechsler, M. Ballauff, and P. Schurtenberger, Giant Hollow Fiber Formation through Self-Assembly of Oppositely Charged Polyelectrolyte Brushes and Gold Nanoparticles, Soft Matter 9, 9111 (2013).

[27] C. L. Phillips, E. Jankowski, M. Marval, and S. C. Glotzer, Self-Assembled Clusters of Spheres Related to Spherical Codes, Phys. Rev. E 86, 041124 (2012).

[28] C. L. Klix, K. I. Murata, H. Tanaka, S. R. Williams, A. Malins, and C.P. Royall, Novel Kinetic Trapping in Charged Colloidal Clusters due to Self-Induced Surface Charge Organization, Sci. Rep. 3, 2072 (2013).

[29] B. G. P. van Ravensteijn and W. K. Kegel, Colloids with Continuously Tunable Surface Charge, Langmuir 30, 10590 (2014).
[30] A. P. Hynninen, C. G. Christova, R. van Roij, A. van Blaaderen, and M. Dijkstra, Prediction and Observation of Crystal Structures of Oppositely Charged Colloids, Phys. Rev. Lett. 96, 138308 (2006).

[31] P. Bartlett and A. I. Campbell, Three-Dimensional Binary Superlattices of Oppositely Charged Colloids, Phys. Rev. Lett. 95, 128302 (2005).

[32] M. Cerbelaud, A. Videcoq, P. Abélard, C. Pagnoux, F. Rossignol, and R. Ferrando, Self-Assembly of Oppositely Charged Particles in Dilute Ceramic Suspensions: Predictive Role of Simulations, Soft Matter 6, 370 (2010).

[33] M. Cerbelaud, A. Videcoq, P. Abélard, C. Pagnoux, F. Rossignol, and R. Ferrando, Heteroaggregation between $\mathrm{Al}_{2} \mathrm{O}_{3}$ Submicrometer Particles and $\mathrm{SiO}_{2}$ Nanoparticles: Experiment and Simulation, Langmuir 24, 3001 (2008).

[34] B. Vincent, M. Jafelicci, P. F. Luckham, and T. F. Tadros, Adsorption of Small, Positive Particles onto Large, Negative Particles in the Presence of Polymer. Part 2. Adsorption Equilibrium and Kinetics as a Function of Temperature, J. Chem. Soc., Faraday Trans. 1 76, 674 (1980).

[35] H. Li, J. Han, A. Panioukhine, and E. Kumacheva, From Heterocoagulated Colloids to Core-Shell Particles, J. Colloid Interface Sci. 255, 119 (2002).

[36] F. Caruso, H. Lichtenfeld, M. Giersig, and H. Möhwald, Electrostatic Self-Assembly of Silica NanoparticlePolyelectrolyte Multilayers on Polystyrene Latex Particles, J. Am. Chem. Soc. 120, 8523 (1998).

[37] M. Kamp, M. Hermes, C. van Kats, D. Kraft, W. Kegel, M. Dijkstra, and A. van Blaaderen (to be published).

[38] M. Raşa, A. P. Philipse, and J. D. Meeldijk, Heteroaggregation, Repeptization and Stability in Mixtures of Oppositely Charged Colloids, J. Colloid Interface Sci. 278, 115 (2004).

[39] D. R. E. Snoswell, T. J. Rogers, A. M. Howe, and B. Vincent, Controlling Porosity within Colloidal Heteroaggregates, Langmuir 21, 11439 (2005).

[40] J. M. López-López, A. Schmitt, A. Moncho-Jordá, and R. Hidalgo-Álvarez, Electrostatic Heteroaggregation Regimes in Colloidal Suspensions, Adv. Colloid Interface Sci. 147-148, 186 (2009).

[41] A. Y. Kim, K. D. Hauch, J. C. Berg, J. E. Martin, and R. A. Anderson, Linear Chains and Chain-like Fractals from Electrostatic Heteroaggregation, J. Colloid Interface Sci. 260, 149 (2003).

[42] E. Sanz, M. E. Leunissen, A. Fortini, A. van Blaaderen, and M. Dijkstra, Gel Formation in Suspensions of Oppositely Charged Colloids: Mechanism and Relation to the Equilibrium Phase Diagram, J. Phys. Chem. B 112, 10861 (2008).

[43] W. Lin, M. Kobayashi, M. Skarba, C. Mu, P. Galletto, and M. Borkovec, Heteroaggregation in Binary Mixtures of Oppositely Charged Colloidal Particles, Langmuir 22, 1038 (2006).

[44] J. M. López-López, A. Schmitt, A. Moncho-Jordá, and R. Hidalgo-Álvarez, Stability of Binary Colloids: Kinetic and Structural Aspects of Heteroaggregation Processes, Soft Matter 2, 1025 (2006).

[45] M. Tagliazucchi, F. Zou, and E. A. Weiss, Kinetically Controlled Self-Assembly of Latex-Microgel Core-Satellite Particles, J. Phys. Chem. Lett. 5, 2775 (2014). 
[46] D. Go, T.E. Kodger, J. Sprakel, and A. J. C. Kuehne, Programmable Co-Assembly of Oppositely Charged Microgels, Soft Matter 10, 8060 (2014).

[47] L. L. Whyte, Unique Arrangements of Points on a Sphere, Am. Math. Mon. 59, 606 (1952).

[48] C. P. Royall, M. E. Leunissen, and A. van Blaaderen, A New Colloidal Model System to Study Long-Range Interactions Quantitatively in Real Space, J. Phys. Condens. Matter 15, S3581 (2003).

[49] A. Yethiraj and A. van Blaaderen, A Colloidal Model System with an Interaction Tunable from Hard Sphere to Soft and Dipolar, Nature (London) 421, 513 (2003).

[50] See Supplemental Material at http://link.aps.org/ supplemental/10.1103/PhysRevX.5.021012 for details of particle syntheses, analyses, characterisations and for movies of permanent clusters and electric field experiments.

[51] M. N. van der Linden, J. C. P. Stiefelhagen, G. HeesselsGrboa, J. E. S. van der Hoeven, N. A. Elbers, M. Dijkstra, and A. van Blaaderen, Charging of Poly(Methyl Methacrylate) (PMMA) Colloids in Cyclohexyl Bromide: Locking, Size Dependence, and Particle Mixtures, Langmuir 31, 65 (2015).

[52] T. Vissers, http://dspace.library.uu.nl/handle/1874/188014.

[53] T. Vissers, A. Wysocki, M. Rex, H. Lowen, C. P. Royall, A. Imhof, and A. van Blaaderen, Lane Formation in Driven Mixtures of Oppositely Charged Colloids, Soft Matter 7, 2352 (2011).

[54] T. Vissers, A. Imhof, F. Carrique, Á. V. Delgado, and A. van Blaaderen, Electrophoresis of Concentrated Colloidal dispersions in Low-Polar Solvents., J. Colloid Interface Sci. 361, 443 (2011).

[55] T. Vissers, A. van Blaaderen, and A. Imhof, Band Formation in Mixtures of Oppositely Charged Colloids Driven by an ac Electric Field, Phys. Rev. Lett. 106, 228303 (2011).
[56] M. Patra, M. Patriarca, and M. Karttunen, Stability of Charge Inversion, Thomson Problem, and Application to Electrophoresis, Phys. Rev. E 67, 031402 (2003).

[57] E. J. W. Verwey and J.T.G. Overbeek, Theory of the Stability of Lyophobic Colloids (Courier Dover Publications, Mineola, New York, 1999).

[58] K. Brown, http://www.mathpages.com/home/kmath005/ kmath005.htm.

[59] Y. S. Cho, G. R. Yi, S. H. Kim, D. J. Pine, and S. M. Yang, Colloidal Clusters of Microspheres from Water-in-Oil Emulsions, Chem. Mater. 17, 5006 (2005).

[60] A. van Blaaderen, Materials Science-Colloids Get Complex, Nature (London) 439, 545 (2006).

[61] A. M. Kalsin, M. Fialkowski, M. Paszewski, S. K. Smoukov, K. J. M. Bishop, and B. A. Grzybowski, Electrostatic SelfAssembly of Binary Nanoparticle Crystals with a Diamondlike Lattice, Science 312, 420 (2006).

[62] A. M. Kalsin and B.A. Grzybowski, Controlling the Growth of "Ionic" Nanoparticle Supracrystals, Nano Lett. 7, 1018 (2007).

[63] B. Liu, T. H. Besseling, M. Hermes, A. F. Demirörs, A. Imhof, and A. van Blaaderen, Switching Plastic Crystals of Colloidal Rods with Electric Fields, Nat. Commun. 5, 3092 (2014).

[64] E. Bianchi, C. N. Likos, and G. Kahl, Tunable Assembly of Heterogeneously Charged Colloids, Nano Lett. 14, 3412 (2014).

[65] M. R. Buck, J. F. Bondi, and R. E. Schaak, A Total-Synthesis Framework for the Construction of High-Order Colloidal Hybrid Nanoparticles, Nat. Chem. 4, 37 (2012).

[66] H. R. Vutukuri, J. Stiefelhagen, T. Vissers, A. Imhof, and A. van Blaaderen, Bonding Assembled Colloids without Loss of Colloidal Stability, Adv. Mater. 24, 412 (2012).

[67] P. Qiu and C. Mao, Viscosity Gradient as a Novel Mechanism for the Centrifugation-Based Separation of Nanoparticles, Adv. Mater. 23, 4880 (2011). 